\title{
A List Based Detection Technique for MIMO Systems
}

\author{
SaiRamesh Nammi
}

Ericsson Inc.

\section{Introduction}

It is well known that multiple transmit and receive antennas can significantly increase the data carrying capacity of wireless systems (1) - (3). Usually a multiple-input multiple-output (MIMO) system requires a channel code to approach capacity (4). However, for such MIMO systems, the optimal joint maximum-likelihood (ML) detection using exhaustive search is too complex and is almost impossible. Motivated by turbo decoding (5), iterative detectors and decoders, treating the channel code as the outer code and the space time mapper as the inner code, have been commonly employed in the literature. Although the iterative decoder structures are extensively investigated in the code theory literature, the design and role of the detector structure still need investigation. In this context, the authors in (6), (9) studied the iterative algorithm for MIMO systems using optimum soft-input/soft-output ML MIMO detector. Unfortunately the optimal MIMO detector complexity increases exponentially with the number of transmit antennas or/and the number of bits per constellation point.

In this chapter, we propose a novel threshold list subset detector (T-LSS) that extends the List subset detector for iterative turbo MIMO systems (20). List based techniques have also been considered by other researchers in various contexts. In (15), a list-sequential detector, based on a modified stack algorithm, is discussed for MIMO systems. Detection over multiple input multiple output channels for uncoded system using Chase (25) type of algorithm has been proposed in (17), and for multiuser detection in spread spectrum systems in (28). The T-LSS detector generates lists of candidate codewords based on the subset sum algorithm (22). The T-LSS detector do not require any search radius as in LSD rather depends on the threshold value. Second, the lists of codewords are generated at each iteration of the receiver by taking into account the a priori information fed back from the channel decoder. Third, the candidate codeword selection in T-LSS does not directly involve channel estimation as in LSD. Our results show that for small average list sizes, the performance of T-LSS is almost same as full $A$ Posteriori Probability (APP) detection. Moreover, the average complexity of T-LSS is relatively small. Using Extrinsic Information Transfer (EXIT) chart analysis, we can design the optimal list size per iteration. Our results indicate that we can reduce the complexity significantly for an iterative MIMO system by using T-LSS in the first iteration and using very low list sizes optimized using EXIT chart.

The rest of the chapter is organized as follows. Section II describes the system model. Section III describes the T-LSS detector structure in the iterative framework. Performance analysis of T-LSS is given in Section IV and Section V discusses the implementation complexity. Selection 


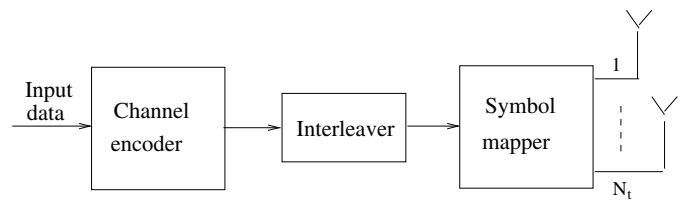

a) Transmitter structure

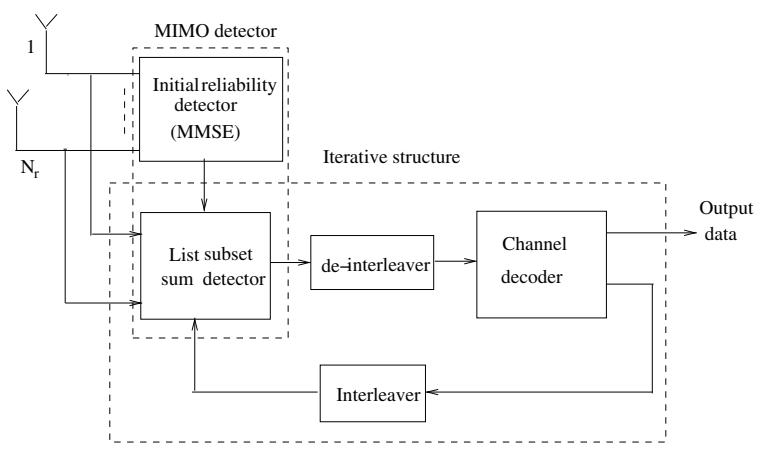

b) Iterative MIMO receiver with T-LSS detector

Fig. 1. Block diagram of turbo MIMO system.

of list sizes using EXIT charts is described in Section VI. Section VII discusses numerical results of T-LSS. Finally, conclusions are presented in Section VIII.

Notation: Bold upper case (lower case) letters denote matrices (vectors). The notations $(\cdot)^{T}$, $(\cdot)^{H},(\cdot)^{*}, E[\cdot], \operatorname{Re}\{\cdot\}$ and $\operatorname{Im}\{\cdot\}$ denote transpose, Hermitian, conjugation, expectation, real and imaginary parts respectively. $A^{c}$ is the complement of $A,\|\cdot\|$ is Euclidian norm, and $\left(\begin{array}{c}m \\ k\end{array}\right)$ denotes $m ! /(m-k) ! k !$.

\section{System description}

Consider a MIMO system with $N_{t}$ transmit antennas and $N_{r}$ receive antennas. At the transmitter side, the input data bits are encoded by a channel code, randomly interleaved and then mapped to modulation symbols before being transmitted by the antennas. Denoting a block of information bits by the vector $\mathbf{d}$ and the transfer function of the channel code by $\mathbf{G}$, the output codeword can be written as $\tilde{\mathbf{c}}=\mathbf{G d}$, and $\mathbf{c}=\Pi(\tilde{\mathbf{c}})$ represents the interleaved sequence of coded bits. The symbol mapper converts this bit sequence into an $\mathrm{M}$-ary symbol sequence as follows. Assume that c has $L N_{t} M_{c}$ elements, where $M_{c}$ is the number of bits per constellation point and $L$ is a non-negative integer. The symbol mapper first partitions c into $L$ subvectors, each of length $N_{t} M_{c}$, to form an $N_{t} M_{c} \times L$ matrix, $\mathbf{C}=\left[\mathbf{c}^{1}, \mathbf{c}^{2} \ldots, \mathbf{c}^{L}\right]$, where $\mathbf{c}^{l}=\left[c_{(l-1) N_{t} M_{c}+1}, c_{(l-1) N_{t} M_{c}+2}, \ldots, c_{l N_{t} M_{c}}\right]^{T}, 1 \leq l \leq L$, and $c_{i}$ is the $i$-th element of c. Each subvector $\mathbf{c}^{l}$ is then mapped to the symbol vector $\mathbf{x}^{l}=\left[x_{1}^{l}, x_{2}^{l}, \ldots, x_{N_{t}}^{l}\right]^{T}$ through a

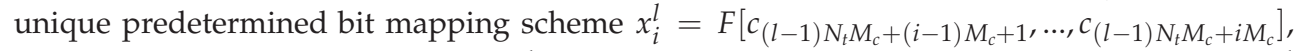
$1 \leq i \leq N_{t}$. We call each sub vector $\mathbf{c}^{l}$ a code bit vector. The elements of the symbol vector $\mathbf{x}^{l}$ are then transmitted by the $N_{t}$ transmit antennas. 
Ignoring the superscript $(l)$ for simplicity, the received $N_{r} \times 1$ vector $\mathbf{r}$ due to the transmission of the $N_{t} \times 1$ symbol vector $\mathbf{x}^{l}$ can be written as

$$
\mathbf{r}=\mathbf{H x}+\mathbf{n}
$$

where $\mathbf{H}$ is a $N_{r} \times N_{t}$ complex MIMO channel matrix, and $\mathbf{n}$ is complex additive noise vector whose elements are independent, complex-valued Gaussian variables with zero mean and variance $\sigma^{2}$ per each dimension.

\section{Soft MIMO detectors}

\subsection{MIMO detector background}

In the literature, different optimal and suboptimal structures have been studied. The suboptimal detectors can be classified into linear and nonlinear detectors. Linear detectors include zero-forcing (ZF) and minimum mean-square error (MMSE) detectors, and the nonlinear receivers include decision feedback, nulling-cancelling and variants relying on successive interference cancellation. These suboptimal detectors are easy to implement but their bit error rate (BER) performance is significantly inferior to that of the optimum MIMO detector (10). The authors in (12), (13) studied nulling-cancelling detector with iterative processing.

There has also been considerable progress in using low complexity (near) ML detection based on lattice decoding. In (19), a near optimal detection method is proposed using sphere-decoder (SD) concept, which has low complexity at high SNR. The work in (4) extends the sphere detector algorithm of (19) to coded MIMO systems with complex constellations. It is shown that for good choices of the initial radius and for sufficient list sizes, list sphere decoder (LSD) in a concatenated system can achieve a bit error rate (BER) performance close to capacity limits. However, the worst case complexity of LSD can be very high and the expected complexity is polynomial with the number of transmit antennas (4). Besides, the LSD does not exploit channel decoder information in generating the list of candidate codewords. Based on semi-definite relaxation (SDR) of the ML problem, the authors in (14) proposed a soft Quasi ML detection for MIMO systems.

\subsection{Conventional soft MIMO detector}

A typical iterative receiver structure consists of an inner MIMO detector and an outer channel decoder. The MIMO detector calculates the log likelihood ratio (LLR) $L_{D}\left(c_{i} \mid \mathbf{r}\right)=\log \left(P\left(c_{i}=\right.\right.$ $\left.1 \mid \mathbf{r}) / P\left(c_{i}=0 \mid \mathbf{r}\right)\right)$, for each bit $c_{i}, 1 \leq i \leq N_{t} M_{c}$, and sends the extrinsic LLR values $L_{\text {ext }}\left(c_{i} \mid \mathbf{r}\right)=$ $L_{D}\left(c_{i} \mid \mathbf{r}\right)-L_{A}\left(c_{i}\right)$ to the channel decoder, where $L_{A}\left(c_{i}\right)=\log \left(P\left(c_{i}=1\right) / P\left(c_{i}=0\right)\right)$ is the $a$ priori information. A direct computation of detector extrinsic LLR is performed as (9)

$$
L_{\mathrm{ext}}\left(c_{i} \mid \mathbf{r}\right)=\log \frac{\sum_{\mathbf{c}^{l} \in \mathrm{E} \cap c_{i, 1}} p\left(\mathbf{r} \mid \mathbf{c}^{l}\right) \cdot \exp \left(\frac{1}{2} \mathbf{c}_{[i]}^{l^{T}} \mathbf{L}_{A,[i]}\right)}{\sum_{\mathbf{c}^{l} \in \mathrm{E} \cap c_{i, 0}} p\left(\mathbf{r} \mid \mathbf{c}^{l}\right) \cdot \exp \left(\frac{1}{2} \mathbf{c}^{\left.l^{T}{ }_{[i]}^{T} \mathbf{L}_{A,[i]}\right)}\right.}
$$

where $€$ is a set containing all possible code bit vectors and is called the list. The set $€ \bigcap c_{i, 1}$ contains code bit vectors in the list with $c_{i}=1,(l-1) N_{t} M_{c}+1 \leq i \leq l N_{t} M_{c}, \mathbf{L}_{A,[i]}$ denotes the vector of all a priori values by omitting the bit $i, \mathbf{c}_{[i]}^{l^{T}}$ denotes the coded bit vector $\mathbf{c}^{l}$ by removing the $i$-th bit, and $p\left(\mathbf{r} \mid \mathbf{c}^{l}\right)$ is the conditional probability density function (pdf). 
From (2), it is clear that an exhaustive search over all possible bit vectors requires computation of (2) over a list $€$ containing $2^{N_{t} M_{c}}$ candidate code bit vectors. This provides MAP detection but its complexity is too high for large $N_{t} M_{\mathcal{C}}$. In order to reduce complexity, the authors in (4) extend the SD concept for coded turbo MIMO systems by generating a list of code bit vectors from the detector to the decoder. Although the complexity is reduced, the list generation process in (4) does not utilize the information fed back from the channel decoder. Accordingly, the work in (34) modifies the SD algorithm with a Finkle-Pohst MAP search, which is repeated for each iteration. In general, the SD based approach relies on a search radius that sometimes involves trial and error (4). Furthermore, many floating point operations, including QR decompositions or Cholesky decompositions, are required in generating the list of candidate code bit vectors. Therefore, we propose a different soft MIMO detector structure that generates lists requiring only a few floating point operations and mostly comparisons. Besides, instead of using a search radius, we use alternative parameters which are easier to characterize.

\subsection{Proposed soft MIMO detector}

In our novel receiver structure, we reduce the list size significantly so that the computation of (2) is performed only for a few candidate bit words. Toward this end, we propose to optimize the list from iteration to iteration. Thus, for the $k$-th iteration between the detector and the decoder, $L_{\text {ext }}^{(k)}\left(c_{i} \mid \mathbf{r}\right)$ is computed as

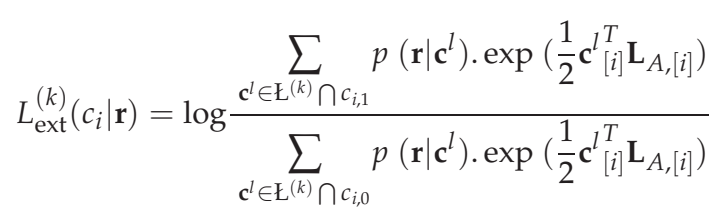

where $\iota^{(k)}$ is the list of code bit vectors in the $k$-th iteration. Note that unlike $2, \epsilon^{(k)}$ does not usually contain all possible code bit vectors. The detector constructs the list based on the LLR information $\left\{\alpha_{i}\right\}$ for each bit. In the first iteration, we use an initial reliability detector to generate $\left\{\alpha_{i}\right\}$. For subsequent iterations, $\left\{\alpha_{i}\right\}$ are obtained from the channel decoder. Thus, our receiver consists of three components : (1) an initial reliability detector, (2) a list subset sum detector and (3) a channel decoder as shown in Fig. 1. The initial reliability detector and the list subset detector form the proposed MIMO detector.

\subsubsection{Initial reliability detector}

The initial reliability detector is used only during the first iteration to assist in the generation of the list $\mathrm{七}^{(1)}$. It consists of a linear minimum mean squared error (MMSE) detector. Such detectors are known to have information lossless property (24). Other detectors namely zero forcing (ZF), zero forcing with successive interference cancellation (ZF-SC), minimum mean squared error with successive interference cancellation (MMSE-SC) can also be used to obtain the initial reliability of bits. Let $\mathbf{y}=\mathbf{P}^{H} \mathbf{r}$ be the $N_{t} \times 1$ vector output of MMSE detector, where $\mathbf{P}=\left(\mathbf{H R}_{x} \mathbf{H}^{H}+\sigma^{2} \mathbf{I}\right)^{-1} \mathbf{H}$ is the MMSE detector coefficients (10), $\mathbf{R}_{x}=E\left[\mathbf{x} \mathbf{x}^{H}\right]$, and $\mathbf{I}$ is the identity matrix of size $N_{r} \times N_{r}$. Assuming MMSE output as Gaussian (21), the MMSE output is used to obtain the initial reliability of the $N_{t} M_{c}$ bits. The reliability of the coded bit $c_{i}$ is measured by the magnitude of the $\log$-likelihood ratio, $\log \left(P\left(c_{i}=1 \mid y_{t}\right) / P\left(c_{i}=0 \mid y_{t}\right)\right)$, where $t=\left\lceil i / M_{\mathcal{C}}\right\rceil$. As an example, for quadrature phase-shift keying (QPSK) with constellation 
points $(1+j) / \sqrt{2},(-1+j) / \sqrt{2},(-1-j) / \sqrt{2},(1-j) / \sqrt{2}$ representing bit pairs $(0,0),(0,1)$, $(1,1)$, and $(1,0)$ respectively, $\operatorname{Im}\left\{y_{t}\right\}$ and $\operatorname{Re}\left\{y_{t}\right\}$ give the reliabilities of the first and the second bit respectively (32). Let us denote the reliability of the coded bits by $\left\{\alpha_{i}\right\}$ and let $\alpha_{i}^{h}$ be the hard decision of $\alpha_{i}$.

\subsubsection{List subset sum detector}

The list subset sum detector takes reliability values $\left\{\alpha_{i}\right\}$ as input soft information to produce a list $\iota^{(k)}$ of most likely code bit vectors. This list is used to produce extrinsic LLR $L_{\text {ext }}\left(c_{i} \mid \mathbf{r}\right)$ for each bit $c_{i}$. It consists of two parts : a list generator and an extrinsic LLR calculator.

List generator: The motivation of list based algorithms is that flipping of $\alpha_{i}^{h}$ at a few bit positions may produce the MAP code bit vector in $\mathrm{E}^{(k)}$ of 3 with high probability. We use the MAP metric of the transmitted vector $\mathbf{c}$, denoted by $J(\mathbf{c})$ as (27)

$$
J(\mathbf{c})=\sum_{i \in S_{c}}\left|\alpha_{i}\right|-\sum_{i \in S_{c}^{n}}\left|\alpha_{i}\right|=\sum_{i \in U}\left|\alpha_{i}\right|-2 \sum_{i \in S_{c}^{n}}\left|\alpha_{i}\right|
$$

where $U=\left\{1,2, \ldots, N_{t} M_{c}\right\}$ is the set of bit positions, $S_{c}=\left\{i \mid c_{i}=\alpha_{i}^{h}, i \in U\right\}$ and $S_{c}^{n}=\left\{i \mid c_{i} \neq\right.$ $\left.\alpha_{i}^{h}, i \in U\right\}$. Since the first term in (4) is independent of the value of $\mathbf{c}, J(\mathbf{c})$ depends only on the second term $\sum_{i \in S_{c}^{n}}\left|\alpha_{i}\right|$. Hence we need to find set of vectors which minimizes the second term. i.e. the perturbation vectors should be designed in ascending order of the sum $S=\sum_{i \in S_{c}^{n}}\left|\alpha_{i}\right|$. That is the problem is equivalent to the subset-sum problem (22), where the objective is to find all subsets of a set of numbers that have sum less than or equal to a given number $V_{\text {th }}$. Let $\mathrm{E}^{(k)}$ be the set of all perturbation vectors whose reliability sum $S$ is below a certain threshold $V_{\text {th }}$ and $\gamma^{(k)}$ be the cardinality of $\iota^{(k)}$. The choice of $V_{\text {th }}$ will be discussed in the next section.

Let us define a vector $\mathbf{p}_{i}$ as a zero element vector with a 1 at the $i$-th bit position. The weight of the vector $\left\{\alpha_{i}\right\}$ is defined as $w=\sum_{i=1}^{N_{t} M_{c}}\left|\alpha_{i}\right|$. The list is generated as follows :

Step 1: Sort $\left|\alpha_{j}\right|, j=1, \ldots, N_{t} M_{c}$ in the ascending order. Set the size of the list $\Lambda=1$, bit position $i=1$, first term in the list $\mathbf{v}_{\text {old }}^{1}=\left[\begin{array}{llll}0 & 0 & 0 . . .0\end{array}\right]$ and the weight $w_{\text {old }}^{1}=0$.

Step 2: For each vector in the list $m=1$ to $\Lambda$, create $\mathbf{v}_{\text {new }}^{m}=\mathbf{v}_{\text {old }}^{m}+\mathbf{p}_{i}$, and modify the weight of each vector as $w_{\text {new }}^{m}=w_{\text {old }}^{m}+\left|\alpha_{i}\right|$.

Step 3: Merge lists $\mathbf{v}_{\text {new }}$ and $\mathbf{v}_{\text {old }}$ to form a new list $\mathbf{v}_{\text {new }}$.

Step 4: If $k=1$ i.e., in the first iteration between the detector and the decoder, keep only the vectors in $\mathbf{v}_{\text {new }}$ that have a sum less than or equal to $V_{\text {th }}$.

Step 5: If $k>1$, then sort vectors in $\mathbf{v}_{\text {new }}$ in ascending order according to their weights $w_{\text {new }}$. Truncate the size of $\mathbf{v}_{\text {new }}$ to $\Lambda^{(k)}$, if the size is greater than $\Lambda^{(k)}$, where $\Lambda^{(k)}$ is the maximum size of the list, and set the value of $\Lambda^{(k)}$ to the size of $\mathbf{v}_{\text {new }}$.

Step 6: Set $\mathbf{v}_{\text {old }} \leftarrow \mathbf{v}_{\text {new. }}$.

Step 7: Set $i \leftarrow i+1$. Repeat Step 2 to 7 if $i \leq N_{t} M_{c}$.

Step 8: Re-arrange the positions of the elements in each test vector in $\mathbf{v}_{\text {new }}$ back to their original positions before Step 1 , and then add each vector to $\alpha^{h}$ to generate the test list $\mathrm{E}^{(k)}$.

Extrinsic LLR calculator: Once the list $\mathrm{E}^{(k)}$ is available, the extrinsic LLR values $L_{\mathrm{ext}}^{(k)}\left(c_{i} \mid \mathbf{r}\right)$ are computed for each coded bit $c_{i}$. For higher number of transmit antennas, computation of (3) is very cumbersome, and max-log approximation is used as (4) 


$$
\begin{aligned}
L_{\mathrm{ext}}^{(k)}\left(c_{i} \mid \mathbf{r}\right) \approx & \frac{1}{2} \max _{\mathbf{c}^{l} \in \mathrm{E}^{(k)} \cap c_{i,+1}}\left\{-\frac{1}{\sigma^{2}}\|\mathbf{r}-\mathbf{H x}\|^{2}+\mathbf{c}_{[i]}^{l^{T}} \mathbf{L}_{A,[i]}\right\} \\
& -\frac{1}{2} \max _{\mathbf{c}^{l} \in \mathrm{E}^{(k)} \cap c_{i, 0}}\left\{-\frac{1}{\sigma^{2}}\|\mathbf{r}-\mathbf{H x}\|^{2}+\mathbf{c}_{[i]}^{l^{T}} \mathbf{L}_{A,[i]}\right\}
\end{aligned}
$$

After all the code bit vectors $c^{l}, 1 \leq l \leq L$, have been processed by the MIMO detector, the extrinsic LLR values associated with $\mathrm{c}$ are made available to the channel decoder as a priori information after de-interleaving:

$$
L^{A}(\tilde{\mathbf{c}})=\Pi^{-1}\left\{L_{\text {ext }}(\mathbf{c})\right\}
$$

\subsection{Channel decoder}

The decoder considered in this chapter is a soft-input soft-output (SISO) convolutional decoder based on the BCJR algorithm (30). This decoder is based on trellis structure, where a branch metric computation uses the soft information $L^{A}(\tilde{\mathbf{c}})$ coming from the detector. Based on forward and reverse recursions of BCJR, the LLR for each coded and data bit is obtained. The a priori information $L^{A}\left(\tilde{c}_{i}\right)$ is removed from the LLR of each coded bit to produce extrinsic information $L^{\text {ext }}\left(\tilde{c}_{i}\right)$. Finally, the extrinsic information is fed back to the detector as a priori information after interleaving as

$$
L_{A}(\mathbf{c})=\Pi\left\{L^{\operatorname{ext}}(\tilde{\mathbf{c}})\right\}
$$

The a priori information $L_{A} c_{i}$ for each bit $c_{i}$ is used by the MIMO detector as described in Section III A. The iteration between the detector and the decoder are repeated to decrease the BER. After the last iteration, the LLR of data bits are subjected to hard decision to produce final data decisions.

\section{BER analysis of MIMO detector}

In this section, we consider BER analysis of the MIMO detector. It is useful for two reasons. First, it directly provides analytical performance results of the proposed detector when used in an uncoded MIMO system. Second, we use this analysis to show selection of $V_{\text {th }}$ used in the list generation algorithm. We follow a procedure similar to (32). However, our final results are different from (32).

We consider QPSK modulation. The probability of an error $e$ in bit $c_{i}$ can be written as

$$
P_{b, i}=\sum_{u=0}^{1} P\left(e \mid c_{i}=u\right) P\left(c_{i}=u\right)
$$

Assume bits 0 and 1 to be equally likely and $P\left(e \mid c_{i}=u\right)$ to be same for $u=0$ and 1 . We can then write $P_{b, i}=P\left(e \mid c_{i}=0\right)$. When the MIMO detector uses $V_{\text {th }}$ to control the list, we can write

$$
\begin{array}{r}
P_{b, i}=P\left(e \mid c_{i}=0, \alpha_{i} \leq V_{\mathrm{th}}\right) P\left(\alpha_{i} \leq V_{\mathrm{th}} \mid c_{i}=0\right) \\
+P\left(e \mid c_{i}=0, \alpha_{i}>V_{\mathrm{th}}\right) P\left(\alpha_{i}>V_{\mathrm{th}} \mid c_{i}=0\right)
\end{array}
$$




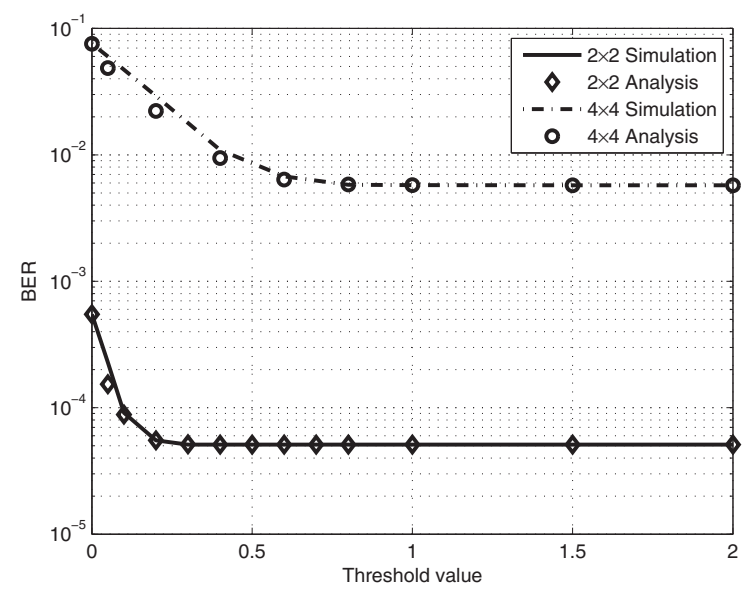

Fig. 2. Effects of $V_{\text {th }}$ on the BER of T-LSS based MIMO system.

Let us denote $P\left(\alpha_{i}>V_{\mathrm{th}} \mid c_{i}=0\right)$ as $P_{\delta, i}$. When $\alpha_{i}>V_{\mathrm{th}}$, the decision on the $\mathrm{i}$-th bit is 1 . Hence $P\left(e \mid c_{i}=0, \alpha_{i}>V_{\mathrm{th}}\right)=1$. We approximate $p\left(\alpha_{i} \mid c_{i}=0\right)$ to be Gaussian with mean $\mu_{i}$ and variance $\sigma_{i}^{2}$. Thus, we get, $P_{\delta, i}=Q\left(\left(V_{\text {th }}+\left|\mu_{i}\right|\right) / \sigma_{i}^{2}\right)$ where $Q($.$) is the Gaussian tail$ probability (10). The evaluation of the term $P\left(e \mid c_{i}=0, \alpha_{i} \leq V_{\text {th }}\right)$ is difficult. However, it can be approximately evaluated as follows.

Consider asymptotically large $V_{\text {th }}$, i.e., $V_{\text {th }} \rightarrow \infty$. In this case, the list contains all possible $2^{N_{t} M_{c}}$ members. Obviously, in this case, $P\left(e \mid c_{i}=0, \alpha_{i} \leq V_{\mathrm{th}} \rightarrow P_{\mathrm{ML}}\right)$, where $P_{\mathrm{ML}}$ is the bit error probability for a maximum likelihood detector. Even for a moderately large $V_{\mathrm{th}}$, since we are interested in the near-ML region of operation, we can still write $P\left(e \mid c_{i}=0, \alpha_{i} \leq V_{\text {th }}\right) \approx P_{\mathrm{ML}}$. Therefore, from (9)

$$
P_{b, i} \approx\left(1-P_{\delta, i}\right) P_{\mathrm{ML}}+P_{\delta, i}
$$

The average BER can be written as

$$
P_{b}=\frac{1}{N_{t} M_{c}} \sum_{i=1}^{N_{t} M_{c}} P_{b, i}=P_{\mathrm{ML}}+\frac{1-P_{M L}}{N_{t} M_{c}} \sum_{i=1}^{N_{t} M_{c}} P_{\delta, i}
$$

We have found this expression to provide accurate results in our extensive simulations. As an example, Figure 2 shows our results for various values of $V_{\mathrm{th}}$. The channel is randomly generated and kept fixed at an SNR of $10 \mathrm{~dB}$. For the $2 \times 2$ system, the rows of the channel matrix is are [1.3-j0.19 0.08-j0.16], and [0.84-j0.09 -0.85-j0.81] respectively. For the $4 \times 4$ system, the rows are $\left[\begin{array}{lllll}0.27-j 0.23 & 0.35-\mathrm{j} 0.21 & 0.21+\mathrm{j} 1.51 & 0.91-\mathrm{j} 0.52\end{array}\right],\left[\begin{array}{llll}0.82+\mathrm{j} 0.46 & 0.40+\mathrm{j} 0.50 & -0.69+\mathrm{j} 0.024\end{array}\right.$ $0.35-\mathrm{j} 1.202]$, [1.16+j0.56 $0.38-\mathrm{j} 0.03 \quad 0.47-\mathrm{j} 0.271 .11+\mathrm{j} 1.25]$, and [-0.02-j0.14 $-0.15-\mathrm{j} 0.360 .69+\mathrm{j} 0.75$ $1.16+j 0.24]$ respectively.

The selection of $V_{\text {th }}$ is done as follows. Suppose we want $P_{b}$ to be within a fraction of $\epsilon$ of $P_{\mathrm{ML}}$, i.e., $P_{b}=(1+\epsilon) P_{\mathrm{ML}}$. From (11), we get

$$
\sum_{i=1}^{N_{t} M_{c}} P_{\delta, i}=\left(\frac{\epsilon P_{\mathrm{ML}}}{1-P_{\mathrm{ML}}}\right) N_{t} M_{c}
$$


which gives

$$
\sum_{i=1}^{N_{t} M_{c}} Q\left(\frac{V_{\text {th }}+\left|\mu_{i}\right|}{\sigma_{i}}\right)=\left(\frac{\epsilon P_{\mathrm{ML}}}{1-P_{\mathrm{ML}}}\right) N_{t} M_{c}
$$

Thus, $V_{\text {th }}$ can be found by solving (13). The parameter $P_{\mathrm{ML}}$ is difficult to obtain (31). We propose to use interference-free error performance $P_{\mathrm{IF}}$ in place of $P_{\mathrm{ML}}$. This is given by

$$
P_{\mathrm{IF}}=\frac{1}{N_{t} M_{c}} \sum_{i=1}^{N_{t} M_{c}} P_{\mathrm{IF}, i}=\frac{1}{N_{t} M_{c}} \sum_{i=1}^{N_{t} M_{c}} Q\left(\sqrt{2 \gamma_{i}}\right)
$$

where $\gamma_{i}$ is the average received SNR for the $\mathrm{i}$-th bit when only the symbol $x_{l}, l=\lceil i / M\rceil$, is present in the system and other symbols are set to zero. Since $P_{\mathrm{IF}} \leq P_{\mathrm{ML}}$, using $P_{\mathrm{IF}}$ for $P_{\mathrm{ML}}$ in (13) ensures an error performance better than or equal to $(1+\epsilon) P_{\mathrm{ML}}$. The parameters $\mu_{i}$ and $\sigma_{i}$ are obtained as described in (32).

\section{Complexity analysis}

In this section we describe the exact number of computations needed for T-LSS detector. The complexity depends on

1. MMSE detector

2. List generator

3. Extrinsic LLR calculator

\subsection{MMSE detector}

Let $N_{v}$ be the number of $V_{\text {th }}$ values where (13) is evaluated. The calculation of $V_{\text {th }}$ requires about $\left(N_{v}+1\right) N_{t} M_{c}+3$ multiplications and $\left(N_{v}+1\right)\left(N_{t} M_{c}-1\right)$ additions. the $\mathrm{Q}$ function values can be obtained from tables or through a combination of tables and a few floating point operations.

Total number of multiplications for computing $\mathbf{H R}_{x} \mathbf{H}^{H}+\sigma^{2} \mathbf{I}$ is equal to $(1 / 2) N_{r}\left(N_{r}+\right.$ 1) $\left(N_{t}+1\right)$ assuming $\mathbf{R}_{x}=\sigma_{s}^{2} \mathbf{I}$, where $\sigma_{s}^{2}=E\left[\left|x_{i}\right|^{2}\right]$. The number of addition operations is equal to $(1 / 2) N_{r}\left(N_{r}+1\right)\left(N_{t}-1\right)+N_{r}$. The number of multiplications and additions for calculating $\left(\mathbf{H R}_{x} \mathbf{H}^{H}+\sigma^{2} \mathbf{I}\right)^{-1} \mathbf{H}$ is equal to $N_{r}^{2} N_{t}$ and $\left(N_{r}-1\right) N_{r} N_{t}$ respectively. The generation of MMSE detector coefficients requires matrix inversion of size $N_{r} \times N_{r}$. From (23), matrix inversion operation requires $N_{r}^{3}$ multiplications and $N_{r}^{3}-2 N_{r}^{2}+N_{r}$ additions. Since the MMSE detector gives reliability of received code vector of $N_{t} M_{c}$ bits, the total number of multiplications per bit is equal to $\left((1 / 2) N_{r}\left(N_{r}+1\right)\left(N_{t}+1\right)+N_{r}^{3}+N_{r}^{2} N_{t}+\left(N_{v}+1\right) N_{t} M_{c}+\right.$ 3) $/ N_{t} M_{c}$ and addition operations is approximately equal to $\left(N_{r}^{3}-2 N_{r}^{2}+2 N_{r}+(1 / 2) N_{r}\left(N_{r}+\right.\right.$ 1) $\left.\left(N_{t}-1\right)+\left(N_{r}-1\right) N_{r} N_{t}+\left(N_{v}-1\right)\left(N_{t} M_{c}-1\right)\right) / N_{t} M_{c}$. Note that in the case of block fading channels, these operations need to be done only once for the whole block. Then the number of operations per bit will decrease depending on the block size.

\subsection{List generator}

The sorting operation in Step 1 is needed over $N_{t} M_{c}$ elements. Hence the total number of comparison operations are $O\left(N_{t} M_{\mathcal{c}} \log _{2}\left(N_{t} M_{\mathcal{C}}\right)\right)$. This can usually be neglected compared to the other comparison operations. Step 2 requires $\sum_{j=1}^{N_{t} M_{c}} 2^{\min \left(j-1, \log _{2} \gamma^{(k)}\right)}=2 \gamma^{(k)}-1+$ $\left(N_{t} M_{c}-\log _{2} \gamma^{(k)}\right) \gamma^{(k)}$ additions, since the list is restricted to a maximum size of $\gamma^{(k)}$, and until that size is reached, the list size doubles for every increase in $i$ value in $\mathbf{p}_{i}$. In Step 4, we compare the sum with $V_{\text {th }}$ for each member in the list. Since the number of 
members doubles for every increase in $i$ value in $\mathbf{p}_{i}$ and half of the members in the list each time have already been compared, we have a worst case number of comparisons as $2 \gamma^{(1)}-1+\left(N_{t} M_{c}-\log _{2} \gamma^{(1)}\right) \gamma^{(1)}$. In Step 5, until $i$ exceeds $\log _{2} \gamma^{(k)}$, only $\left(2^{i}-1\right)$ comparisons are needed for a given $i$. This is due the special structure of the algorithm where the members after merging in Step 3 remain in the form of two sorted lists. Therefore, the cumulative number of comparisons till $i$ exceeds $\log _{2} \gamma^{(k)}$ can be obtained by summing $\left(2^{i}-1\right)$ over $i$. Defining $\gamma^{(k)^{\prime}}=2^{n}$, where $n=\left\lceil\log _{2} \gamma^{(k)}\right\rceil$, the number of comparisons is about $2 \gamma^{(k)^{\prime}}-\log _{2} \gamma^{(k)^{\prime}}$. When $i>\log _{2} \gamma^{(k)}$ then the list doubles (after Step 3) for every increase in the $i$ value, but members remain in two sorted lists. We thus need to select $\gamma^{(k)}$ elements from $2 \gamma^{(k)}$ elements available in two sorted lists. Thus, $\gamma^{(k)}$ comparisons are needed for each $i$ when $i>\log _{2} \gamma^{(k)}$. Thus, Step 5 requires $2 \gamma^{(k)^{\prime}}-\log _{2} \gamma^{(k)^{\prime}}+\left(N_{t} M_{c}-\right.$ $\left.\log _{2} \gamma^{(k)}\right) \gamma^{(k)}$ comparisons. In summary, if $\eta$ iterations are used, the total number of additions per bit is approximately $\left(1 /\left(N_{t} M_{c}\right)\right) \sum_{k=1}^{\eta}\left(2 \gamma^{(k)}-1+\left(N_{t} M_{c}-\log _{2} \gamma^{(k)}\right) \gamma^{(k)}\right)$. The total number of comparisons per bit is about $\eta \log _{2}\left(N_{t} M_{c}\right)+\left(1 /\left(N_{t} M_{c}\right)\right)\left(2 \gamma^{(1)}-1+\left(N_{t} M_{c}-\right.\right.$ $\left.\left.\log _{2} \gamma^{(1)}\right) \gamma^{(1)}\right)+\left(1 /\left(N_{t} M_{c}\right)\right) \sum_{k=2}^{\eta}\left(2 \gamma^{(k)^{\prime}}-\log _{2} \gamma^{(k)^{\prime}}+\left(N_{t} M_{c}-\log _{2} \gamma^{(k)}\right) \gamma^{(k)}\right)$.

\subsection{Extrinsic LLR calculator}

The extrinsic LLR values are calculated using (5). We can see that $\mathbf{r}-\mathbf{H x}$ requires $N_{r} N_{t}$ multiplications and $N_{r} N_{t}$ addition operations for each member in the list. The norm operation $\|\mathbf{r}-\mathbf{H x}\|^{2}$ requires $N_{r}$ multiplications and $N_{r}-1$ additions. Multiplication by $1 / \sigma^{2}$ requires 1 multiplication operation. The term $\mathbf{c}_{[i]}^{l^{T}} \mathbf{L}_{A,[i]}$ require $N_{t} M_{\mathcal{C}}-2$ addition operations and the summation of the two terms require 1 addition operation. There are $\gamma^{(k)}$ comparison operations. Thus there are $\frac{1}{N_{t} M_{c}}\left(N_{r} N_{t}+N_{r}+1\right) \sum_{k=1}^{\eta} \gamma^{(k)}$ multiplications per bit, $\frac{1}{N_{t} M_{c}}\left(N_{r} N_{t}+N_{t} M_{c}+N_{r}-2\right) \sum_{k=1}^{\eta} \gamma^{(k)}$ additions per bit and $\frac{1}{N_{t} M_{c}} \sum_{k=1}^{\eta} \gamma^{(k)}$ comparisons per bit.

\section{Iterative list detection for turbo MIMO systems}

For MIMO systems concatenated with a channel code, the list size is controlled using $V_{\text {th }}$ during the first iteration. Equation (5) is used to generate extrinsic LLR values that are passed to the channel decoder via the de-interleaver. Since $\mathrm{t}^{(k)}$ does not contain all the code bit vectors, it may so happen for a few bits that they are either 0 or 1 throughout the list. In these cases, we can set the LLR of that particular bit to some fixed value such as +8 or -8 depending on the bit reliability, or we can use the bit flipping algorithm as in (28). In bit flipping method, there is a list size penalty. Once we obtain the channel decoder output information of all the coded bits, they are passed through the interleaver to the MIMO detector. Since the channel decoder outputs are more reliable than the initial reliability detector, the detector uses them for subsequent iterations. In that case, the list size can simply be fixed at the value provided by the first iteration. Fixed list sizes have also been considered in (20). However, further reduction in list sizes can be obtained using an EXIT chart as described below.

\subsection{List detector transfer curves}

The mutual information transfer curve of List MIMO detector can be computed by assuming a priori information $L$ from the channel decoder to be Gaussian distributed with variance $\sigma_{A}^{2}$ and mean $\sigma_{A}^{2} / 2$. The measure of information is computed between the a priori LLR and the 
true transmitted bits as well as between extrinsic output LLR and the transmitted bits. The mutual information at the input of the MIMO detector is denoted as $I_{A_{1}}$ and at the output as $I_{E_{2}}$. The mutual information between transmitted bit $x$ and the LLR $L$ is given by (35), (36)

$$
\begin{array}{r}
I(B, L)=\frac{1}{2} \sum_{d_{i}=-1,1} \int_{-\infty}^{\infty} p(l \mid B=b) \times \\
\log _{2} \frac{2 p(l \mid B=b)}{p(l \mid B=-1)+p(l \mid B=1)} d l
\end{array}
$$

The measure of a-priori information is characterized by $\sigma_{A}^{2}$. The value $\sigma_{A}$ for that particular measure of input mutual information $I_{A}$ is calculated using the relationship

$$
J(\sigma)=I_{A_{1}}\left(\sigma_{A}=\sigma\right)
$$

where

$$
J\left(\sigma_{A}\right)=1-\int_{-\infty}^{\infty} \frac{e^{-\left(l-\sigma_{A}^{2} / 2\right)^{2} / 2 \sigma_{A}^{2}}}{\sqrt{2 \pi \sigma_{A}^{2}}} \log _{2}\left(1+e^{-l}\right) d l
$$

For every given value of $I_{A}$, the MIMO detector is simulated to obtain histogram based pdf, which is used in 15 to compute the mutual output information $I_{E_{1}}$.

\subsection{List optimization using mutual information approach}

Since we would like to ensure convergence, the EXIT optimization is done under two worse scenarios: (1) A low SNR value is used so that the list size found from the chart also works at high SNR. In practice, charts pre-computed at several SNR values can be used. (2) The EXIT chart assumes that extrinsic LLR is used both in the list generator as well as in the extrinsic LLR calculator. This is required to simplify the EXIT plots. This, however, is a worse scenario compared to the actual algorithm where full LLR is used in the list generator while extrinsic LLR is used in the extrinsic LLR calculator.

Fig. 3 shows the EXIT characteristics for the MIMO detector using T-LSS and a rate 1/2 convolutional decoder with constraint length 7 with polynomials [147, 117]. From the numerical simulations, the average list size for T-LSS is found to be 35 . This is the list size in the first iteration. For subsequent iterations, the figure shows that the list size can be reduced to 32 and 24, that can provide performance very close to a full list with 256 members.

Fig. 4 shows the EXIT characteristics for the case of $N_{t}>N_{r}$, In this case, the average list size for the T-LSS detector is 81 . The list size can be reduced to 48 and then to 32 for the second and higher iterations. This can reduce numerical complexity significantly.

\section{Numerical Results and Discussions}

A MIMO system with $N_{t}$ transmit and $N_{r}$ receive antennas is considered. We use a complex Gaussian random MIMO channel. In our work, the channel changes for each code bit vector although our technique applies to block fading channels as well. The channel information is assumed to be known at the receiver only. QPSK modulation is considered for all the cases. Unless specified, $\epsilon$ is set to 0.01 .

Fig 5 shows the BER plot of the MIMO detector (uncoded system) with symmetrical configurations for $2 \times 2,4 \times 4,6 \times 6$ and $8 \times 8$ systems. Full search maximum likelihood detector (MLD) results are also shown. It can be seen that with T-LSS we can reach the 


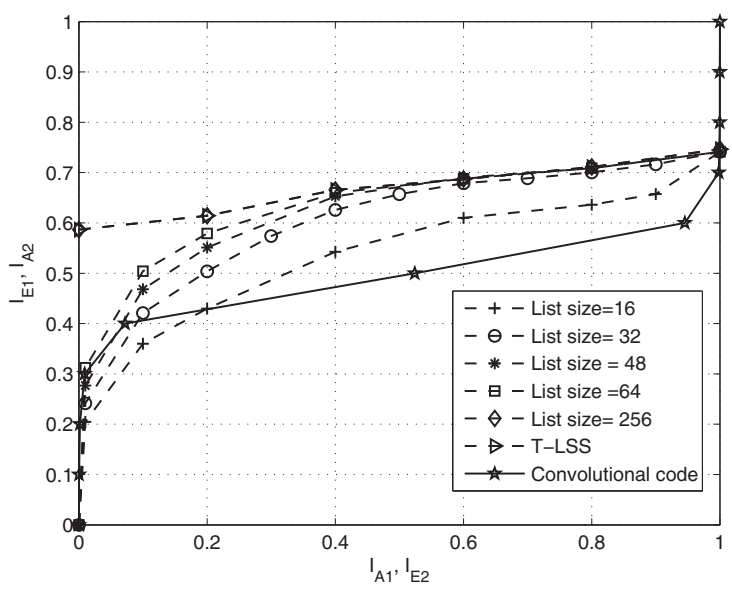

Fig. 3. EXIT characteristics for $4 \times 4 \mathrm{MIMO}$ system with LSS detector at $E_{b} / N_{0}=4.0 \mathrm{~dB}$. Note that average complexity for T-LSS is 35 .

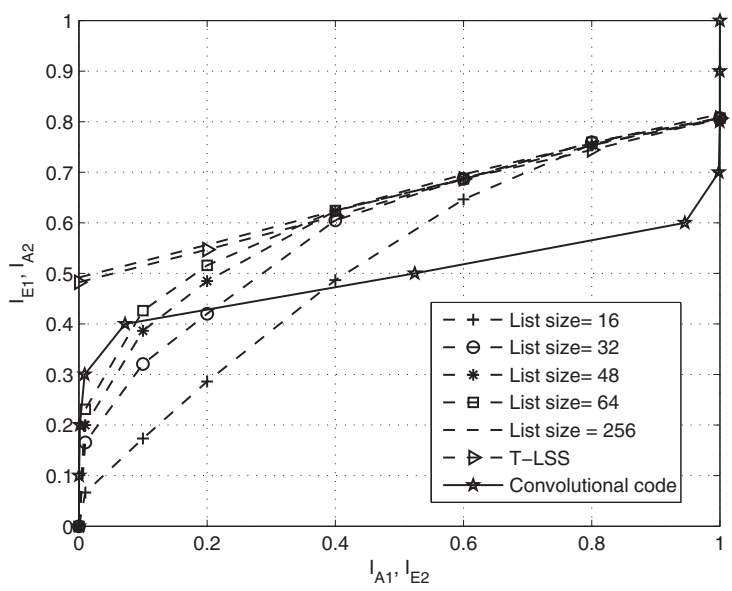

Fig. 4. EXIT characteristics for $4 \times 2 \mathrm{MIMO}$ system with T-LSS detector at $E_{b} / N_{0}=6.0 \mathrm{~dB}$. Note that average complexity for T-LSS is 81 . 
same performance as that of MLD. The average list $S_{\text {avg }}$ required for T-LSS symmetrical configuration is shown in Fig 6. We observe that we can get significant reduction in the list size at high $E_{b} / N_{o}$. For example, for a $4 \times 4$ system at $E_{b} / N_{o}=14 d B$, the average list size equal to 18 , as compared to 256 for MLD.

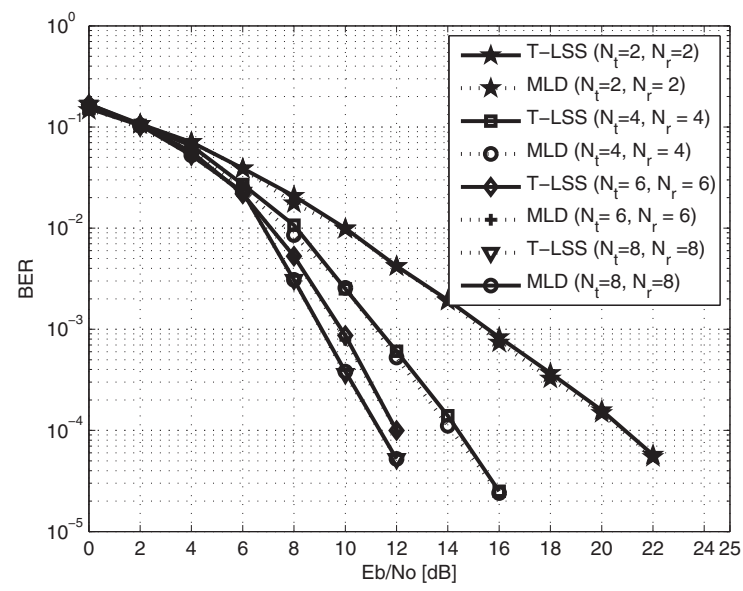

Fig. 5. BER performance of T-LSS for symmetrical configuration.

Fig 7 shows the BER results for a $4 \times N_{r}$ system for different values of $N_{r}$. We note that the T-LSS detector works for any of the combinations of $N_{t}$ and $N_{r}$ unlike the SD, or the original vertical Bell Labs layered space-time (V-BLAST) algorithm. Fig 8 shows the average list sizes of the T-LSS for these configurations. Reduction in the number of receiver antennas results in a loss in diversity and, therefore, an equivalent SNR loss. Therefore, the benefit does

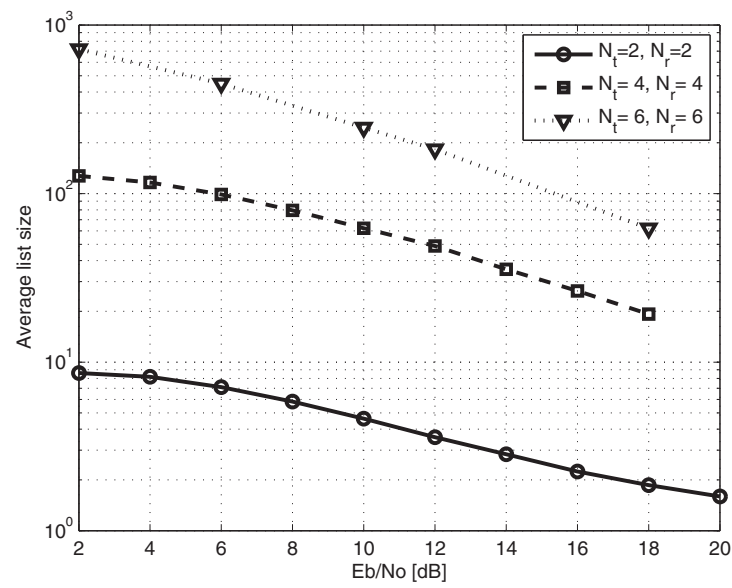

Fig. 6. Average list size per bit of T-LSS for symmetrical configuration. 
not significantly improve with an increase in SNR in the low SNR region. Nevertheless, the average list size for the $4 \times 2$ system is reduced to 90 in contrast with the ML size of 256 .

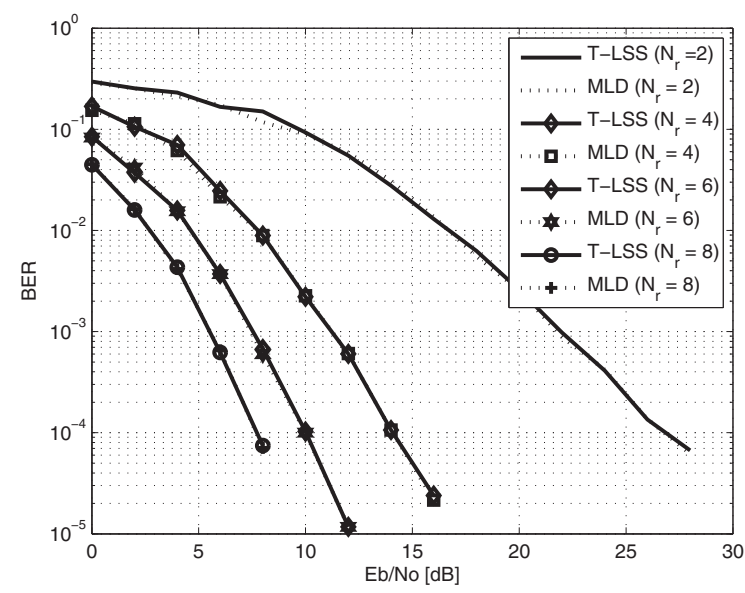

Fig. 7. BER performance of T-LSS for $4 \times N_{r}$ systems.

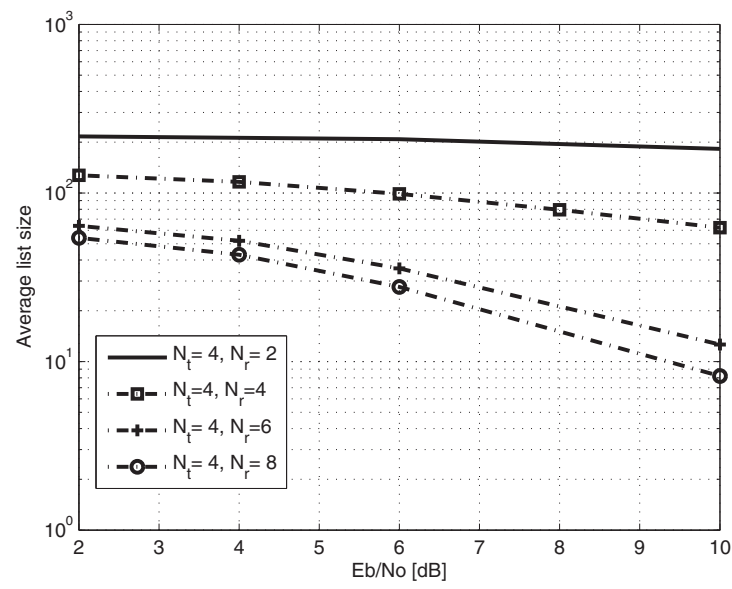

Fig. 8. Average list size per bit of T-LSS for $4 \times N_{r}$ systems.

The role of the parameter $\epsilon$ on the average list size for a $4 \times 4$ system is shown in Fig 9. Note that to get exact ML performance, we need a list size of 256 in this case. However, by relaxing the requirement to $(1+\epsilon) P_{\mathrm{ML}}$, the list size is significantly reduced. Thus at a $15 \mathrm{~dB} \mathrm{SNR}$, we reduce the list size 17 times by targeting for a BER of $1.01 P_{\mathrm{ML}}$ instead of exact $P_{\mathrm{ML}}$. The complexity reduction depends on the SNR.

Fig. 10 shows the BER plot for the iterative MIMO system. A convolutional code with polynomials as described in Section VI is considered. In the first iteration, the list is generated from the T-LSS algorithm. Subsequent iterations use $\gamma^{(k)}$ obtained from the EXIT chart at 4.0 
$\mathrm{dB}$ (Fig 3). We observe that the BER performance of the proposed method closely matches with full search MLD where a list size of 256 is used. For the proposed method, using list sizes of 35, 32, 24 and 24 respectively in the four iterations, the number of multiplications per bit is about 420 using $N_{v}=100$. For the full list MLD, the number of multiplications per bit is about 2688 that comes from the extrinsic LLR calculator. For larger number of antennas or constellation sizes, the potential benefit in complexity will be much more significant.

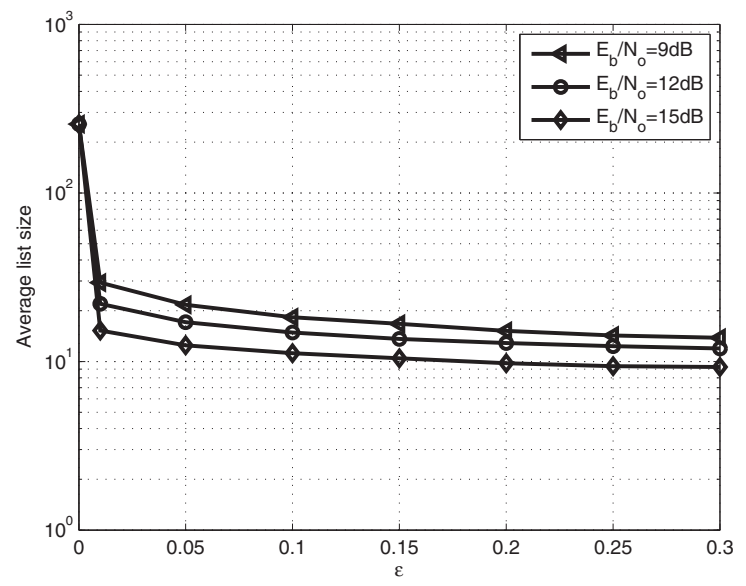

Fig. 9. Effects of $\epsilon$ on the complexity.

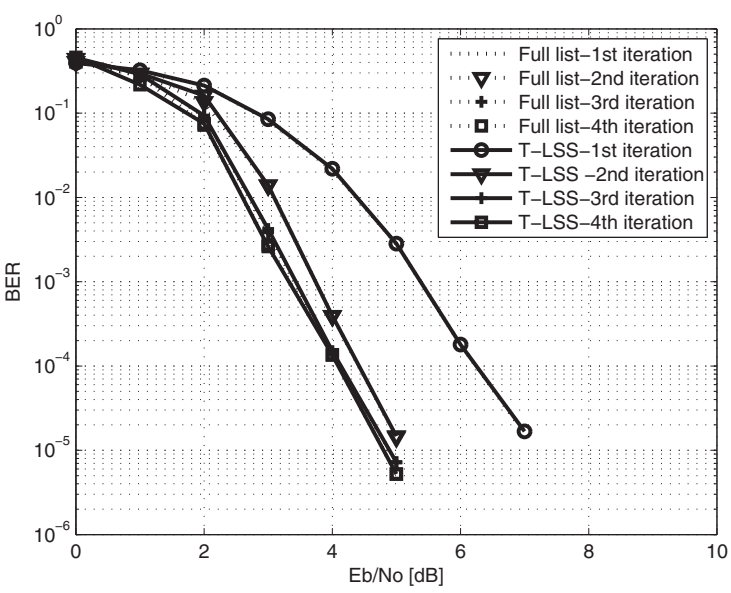

Fig. 10. BER performance of iterative T-LSS for $4 \times 4$ MIMO system.

\section{Conclusions}

A soft input soft output MIMO detector for turbo MIMO systems is presented. The detector searches over a list of candidate codewords to find the most likely codeword vector. The 
BER performance of the detector is analyzed, and a method to choose the optimal list size is described. During the first iteration, the detector uses a threshold value to limit the number of list members, while subsequent iterations use list sizes obtained through EXIT analysis. The numerical complexity of the detector is analyzed. We show that the detector provides BER performance very close to the use of ML detector based receiver at significantly low complexity. Possible future work includes design of capacity approaching irregular LDPC codes based on the proposed T-LSS detector.

\section{References}

[1] I. E. Telatar, "Capcity of multi-antenna Gaussian channels," Eur. Trans. Telecommun., vol. 10, pp. 585-595, Nov. 1999.

[2] G. D. Golden, G. J. Foschini, R. A. Valenzuela, and P. W. Wolnianski, “Detection algorithm and initial laboratory results using the V-BLAST space-time communication architecture," Electron. Lett., vol. 35, pp. 14-15, Jan. 1999.

[3] G. J. Foschini, "Layered space-time architecture for wireless communication in a fading environment when using multi-element antennas," Bell Labs. Tech. J., vol. 1, pp. 41-59, Feb. 1996.

[4] B. M. Hochwald and S. T. Brink, "Achieving near-capacity on a multiple-antenna channel," IEEE Trans. Commun., vol. 51, pp. 389-399, Mar. 2003.

[5] C. Berrou, A. Glavieux, and P. Thitimajshima, "Near Shannon limit error correcting coding and decoding: turbo codes," in Proc. IEEE ICC, Geneva,Switzerland, pp. 1064-1070, May 1993.

[6] A. M. Tonello, "Space-time bit-interleaved coded modulation with an iterative decoing strategy," in Proc. IEEE VTC'2000, pp. 473-478, Boston, May 2001.

[7] S. T. Brink, J. Speidel, and R. H. Yan, "Iterative demapping and decoding for multilevel modulation," in Proc. IEEE GLOBECOM' 1998, pp. 579-584, Sydney, Nov 1998.

[8] J. Hagenauer, E. Offer, and L. Papke, "Iterative decoding of binary block and convolutional codes," IEEE Trans. Inform. Thoery, vol. 42, pp.429-445, March 1996.

[9] A. V. Zelst, R. V. Nee, and G. Awater, "Turbo-BLAST and its performance," in Proc. IEEE VTC' 2001, pp. 1282-1286, Greece, May 2001.

[10] J. G. Proakis, Digital Communications, 4th ed. New York: McGraw-Hill, 2000.

[11] R. D. Maddock and A. H. Banihashemi, "Reliability-based coded modulation with low-density parity-check codes," IEEE Trans. Commun., vol. 54, pp. 403-406, Mar. 2006.

[12] M. Sellathurai and S. Haykin, "Turbo-BLAST for wireless communications: theory and experiments, "IEEE Trans. Signal Processing, vol. 50, pp. 2538-2546, Oct. 2002.

[13] S. L. Ariyavisitakul, "Turbo space-time processing to improve wireless channel capacity," IEEE Trans. Commun., vol. 48, pp. 1347-1359, Aug 2000.

[14] B. Steingrimsson, Z. Q. Luo and K. M. Wong, "Soft quasi maximum likelihood detection for multiple antenna wireless channels, "IEEE Trans. Signal Processing, vol. 51, pp. 2710-2719, Nov. 2003.

[15] S. Baro, J. Hagenauer, and M. Witzke, "Iterative detection of MIMO transmission using a list-sequential (LISS) detector," Proc. ICC'2004, Seattle, 2003.

[16] D. Chase, "A class of algorithms for decoding block codes with channel measurement information," IEEE Trans. Inform. Theory, vol. 18, pp. 170-182, Jan. 1972.

[17] D. Waters and J. R. Barry, "The Chase family of detection algorithms for multiple-input multiple-output channels," Proc. IEEE GLOBECOM'2004, Dallas, 2004. 
[18] Z. Qin and K. C. Teh, "Iterative reduced-complexity multiuser detection based on Chase decoding for synchronous turbo-coded CDMA system," IEEE J. Select. Areas Commun., vol. 24, pp. 200-208, Jan. 2006.

[19] E. Viterbo and J. Boutros, "A universal lattice code decoder for fading channels," IEEE Trans. Inform. Theory, vol. 45, pp. 1639-1642, July 1999.

[20] S. Nammi and D. K. Borah, "Iterative List-Subset Detectors for Turbo Product Coded MIMO Wireless Systems," Proc. IEEE VTC'2007, Dublin, 2007.

[21] H. V. Poor and S. Verdu, "Probability of error in MMSE multiuser detection," IEEE Trans. Inform. Theory, vol. 43, pp. 858-871, May 1997.

[22] T. H. Cormen, C. E. Leiserson, and R. L. Rivest, Introduction to algorithms, 4th ed. New York: McGraw-Hill, 1990.

[23] R. L. Burden, J. D. Faires, and A. C. Reynolds, Numerical analysis, 2nd ed. Boston: Prindle, Weber and Schmid, 1981.

[24] D. Tse and P. Viswanath, Fundamentals of Wireless Communication, Cambridge University Press, 2005.

[25] D. Chase, "A class of algorithms for decoding block codes with channel measurement information," IEEE Trans. Inform. Theory, vol. 18, pp. 170-182, Jan. 1972.

[26] R. Pyndiah, "Near-optimum decoding of product codes: Block turbo codes," IEEE Trans. Commun., vol. 46, pp. 1003-1010, Aug 1998.

[27] T. Kaneko, T. Nishijima, H. Inazumi, and S. Hirasawa, "An efficient maximum-likelihood-decoding algorithm for linear block codes with algebraic decoder," IEEE Trans. Inform. Theory, vol. 40, pp. 320-327, March 1994.

[28] Z. Qin and K. C. Teh, "Iterative reduced-complexity multiuser detection based on chase decoding for synchronous turbo-aided CDMA system," IEEE J. Select. Areas Commun., vol. 24, pp. 200-208, Jan. 2006.

[29] E. Zimmermann, W. Rave, and G. Fettweis, "On the complexity of sphere decoding," Proc. WPMC'2004, Italy, 2004.

[30] L. R. Bahl, J. Cocke, F. Jelinek, and J. Raviv, “Optimal decoding of linear codes for minimizing symbol error rate," IEEE Trans. Inform. Theory, vol. 20, pp. 284Ü287, March 1974.

[31] X. Zhu, and R. D. Murch, " Performance analysis of maximum likelihood detection in a MIMO antenna system," IEEE Trans. Commun., vol. 50, pp. 187-191, Feb. 2002.

[32] S. Nammi and D. K. Borah, "List-based detection in fading channels with long intersymbol interference," IEEE Trans. Wireless Commun., vol. 8, pp. 1276-1283, March 2009.

[33] K. R. Narayanan, X. Wang and G. Yue "Estimating the PDF of the SIC-MMSE equalizer output and its applications in designing LDPC codes with turbo equalization," IEEE Trans. Wireless Commun., vol. 4, pp. 278-287, Jan. 2005.

[34] H. Vikalo, B. Hassibi and T. Kailath, "Iterative decoding of MIMO channels via modified sphere decoder," IEEE Trans. Wireless Commun., vol. 3, pp. 2299-2311, 2004.

[35] S. T. Brink, G. Kramer, and A. Ashikhmin, "Design of low-density parity check codes for modulation and detection," IEEE Trans. Commun., vol. 52, pp. 670-678, April 2004.

[36] S. T. Brink, "Convergence behavior of iteratively decoded parallel concatenated codes," IEEE Trans. Commun., vol. 49, pp. 1727-1737, Oct. 2001. 


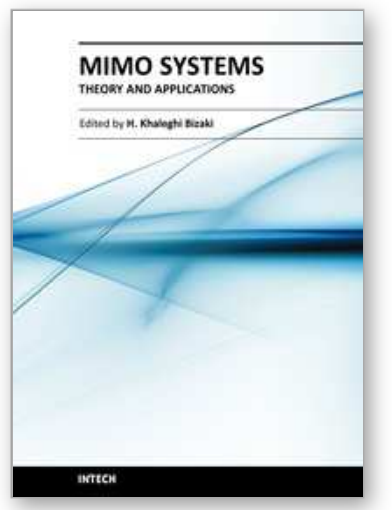

\author{
MIMO Systems, Theory and Applications \\ Edited by Dr. Hossein Khaleghi Bizaki
}

ISBN 978-953-307-245-6

Hard cover, 488 pages

Publisher InTech

Published online 04, April, 2011

Published in print edition April, 2011

In recent years, it was realized that the MIMO communication systems seems to be inevitable in accelerated evolution of high data rates applications due to their potential to dramatically increase the spectral efficiency and simultaneously sending individual information to the corresponding users in wireless systems. This book, intends to provide highlights of the current research topics in the field of MIMO system, to offer a snapshot of the recent advances and major issues faced today by the researchers in the MIMO related areas. The book is written by specialists working in universities and research centers all over the world to cover the fundamental principles and main advanced topics on high data rates wireless communications systems over MIMO channels. Moreover, the book has the advantage of providing a collection of applications that are completely independent and self-contained; thus, the interested reader can choose any chapter and skip to another without losing continuity.

\title{
How to reference
}

In order to correctly reference this scholarly work, feel free to copy and paste the following:

SaiRamesh Nammi (2011). A List Based Detection Technique for MIMO Systems, MIMO Systems, Theory and Applications, Dr. Hossein Khaleghi Bizaki (Ed.), ISBN: 978-953-307-245-6, InTech, Available from:

http://www.intechopen.com/books/mimo-systems-theory-and-applications/a-list-based-detection-technique-formimo-systems

\section{INTECH}

open science | open minds

\section{InTech Europe}

University Campus STeP Ri

Slavka Krautzeka 83/A

51000 Rijeka, Croatia

Phone: +385 (51) 770447

Fax: +385 (51) 686166

www.intechopen.com

\section{InTech China}

Unit 405, Office Block, Hotel Equatorial Shanghai

No.65, Yan An Road (West), Shanghai, 200040, China 中国上海市延安西路65号上海国际贵都大饭店办公楼 405 单元

Phone: +86-21-62489820

Fax: $+86-21-62489821$ 
(C) 2011 The Author(s). Licensee IntechOpen. This chapter is distributed under the terms of the Creative Commons Attribution-NonCommercialShareAlike-3.0 License, which permits use, distribution and reproduction for non-commercial purposes, provided the original is properly cited and derivative works building on this content are distributed under the same license. 